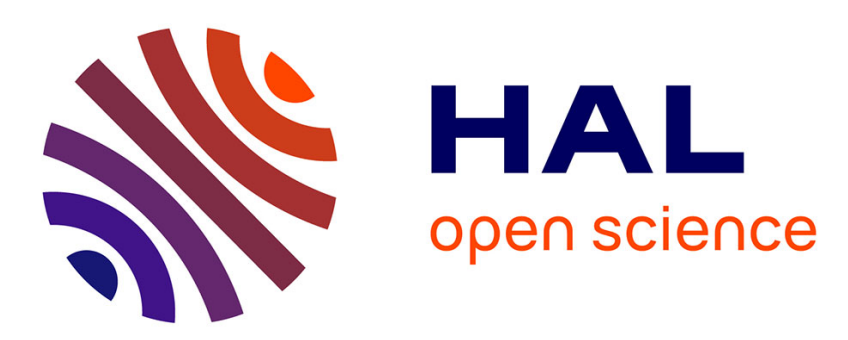

\title{
Reachability-based feedback control of crystal size distribution in batch crystallization processes
}

K. Zhang, M. Nadri, Cheng-Zhong Xu

\section{To cite this version:}

K. Zhang, M. Nadri, Cheng-Zhong Xu. Reachability-based feedback control of crystal size distribution in batch crystallization processes. Journal of Process Control, 2012, 22 (10), pp.1856-1864. 10.1016/j.jprocont.2012.10.002 . hal-02132074

\section{HAL Id: hal-02132074 \\ https://hal.science/hal-02132074}

Submitted on 4 Dec 2019

HAL is a multi-disciplinary open access archive for the deposit and dissemination of scientific research documents, whether they are published or not. The documents may come from teaching and research institutions in France or abroad, or from public or private research centers.
L'archive ouverte pluridisciplinaire $\mathbf{H A L}$, est destinée au dépôt et à la diffusion de documents scientifiques de niveau recherche, publiés ou non, émanant des établissements d'enseignement et de recherche français ou étrangers, des laboratoires publics ou privés. 


\title{
Reachability-based feedback control of crystal size distribution in batch crystallization processes
}

\author{
K. Zhang ${ }^{\mathrm{a}, \mathrm{b}}$, M. Nadri ${ }^{\mathrm{a}, \mathrm{b}}$, C.-Z. Xu ${ }^{\mathrm{a}, \mathrm{b}, *}$ \\ a Université de Lyon, F-69622, Lyon, France \\ b Université Lyon 1, LAGEP, CNRS UMR 5007, 43 Boulevard du 11 novembre 1918, F-69622 Villeurbanne, France
}

\section{A R T I C L E I N F O}

\section{Article history:}

Received 8 November 2011

Received in revised form 7 September 2012

Accepted 11 October 2012

Available online 9 November 2012

\section{Keywords:}

Reachability analysis

Population balance

Crystal size distribution

Hyperbolic partial differential equations

Output feedback control

Batch crystallization process

Observer design

\begin{abstract}
A B S T R A C T
In the paper, we investigate the controllability of crystallization processes by reachability analysis. Crystallization processes are governed by hyperbolic partial differential equations. Given a desired crystal size distribution, we study its reachability by the temperature control from the initial condition without seeding. When the desired crystal size distribution is reachable, we construct an admissible control steering the state to the desired distribution. Our construction is developed based on the discretized model. To ensure that the desired distribution be reached facing model uncertainty, we propose an output feedback control law to correct errors resulted from disturbed parameters of the model.
\end{abstract}

(c) 2012 Elsevier Ltd. All rights reserved.

\section{Introduction}

In many industries, crystallization is the most common way for producing high value chemicals with high purity, desired sizes and desired shapes. It is usually used by separation and purification processes in pharmaceutical, chemical and food industries. The mathematical description of crystallization processes is essentially based on the population balance equations (PBE), coupled with integral differential algebraic equations. The obtained dynamical models are described by a first order partial differential equation (PDE) in which the important state variable, crystal size distribution (CSD), depends on both time and crystal size. The CSD determines the quality of the crystallization products, and it also affects the production efficiency of the subsequent products when the crystallization products are the semi-products. Hence we have for the main objective to develop a method of controlling the crystallizer state to obtain the desired CSD. In the present work, our study is focused on the batch cooling crystallization.

Recently many control problems have been investigated for batch cooling crystallization (cf. [1,2]). Among these studies, two main directions have been identified: the first is based on the

\footnotetext{
* Corresponding author at: Université Lyon 1, LAGEP, CNRS UMR 5007, 43 Boulevard du 11 novembre 1918, F-69622 Villeurbanne, France. Tel.: +33 4724318 90; fax: +33472431899.

E-mail address: xu@lagep.univ-lyon1.fr (C.-Z. Xu).
}

robustness analysis, which mainly deals with the impact of disturbances and model uncertainty on the quality of control strategies (see $[3,4])$; the second is the optimization approach, which studies and optimizes some crystallization property factors such as the mean crystal size, the ratio between nucleated crystal mass and seed crystal mass (see [5-7]). Meanwhile the two approaches have been combined together to solve the optimization problem facing model uncertainty (see $[8,9]$ ).

To design a control scheme for a given system, studying controllability is an important step. The controllability analysis is a necessary expedient to the control design and may help us to develop wise control strategies. However there exist few studies on the controllability of crystallization processes. In [10], Semino and Ray have investigated the controllability of the PBE and demonstrated some controllability results for a continuous crystallizer controlled by using feed concentration without constraint. In [11] the controllability of the feed-batch Draft-Tube crystallizer has been examined by using the evaporation of solvent. Vollmer and Raisch have tackled the controllability problem of batch crystallization with a system inversion approach and gave a feed-forward control [12]. Their approach consists to work essentially on a finite-dimensional moment model. The approach that we consider here is essentially based on the method of characteristics for the hyperbolic PDE model. We have been inspired by the work of Li and Rao [13]. Therefore the controllability problem remains an interesting and open problem for general crystallization processes. 
The present paper deals with the controllability of a batch cooling crystallization process using the temperature control. We like to know if a given crystal size distribution is reachable by the temperature control. When the desired crystal size distribution is reachable, we like to know how to construct an admissible temperature control strategy steering the state to the desired distribution. To do so, we use the reachability analysis carried out in the first step, and we are able to plan a temperature control trajectory to achieve the defined objective. The control design resorts to a feedback control scheme obtained by the reachability analysis. Furthermore another feedback control law is added to the closed-loop system to compensate the model uncertainties. To make possible the application of our feedback control laws, we provide a simple high-gain observer to estimate the zeroth moment which is an unknown quantity of the control laws.

The paper is organized as follows. In Section 2, the batch crystallization model will be presented. Section 3 gives a discretized model with the method of characteristics (MOC). In Section 4, the reachability of desired CSD for a batch cooling crystallization will be studied. Based on the reachability analysis, feedback control laws will be proposed, by taking into account nucleation parameter uncertainty, to achieve targeted CSD facing perturbed parameters. To realize the proposed feedback control, a high gain observer is designed to estimate the zeroth moment which is an unknown quantity of the control law. In Section 6, numerical simulation results will be presented to illustrate the performances of the proposed control strategies. The last section is devoted to our conclusions.

\section{Dynamical models of batch crystallization}

The considered model is based on the ideal batch cooling crystallizer on supersaturation, where breakage and aggregation of crystals are neglected $[14,15]$. The growth rate is assumed to be independent of crystal size according to the McCabe hypothesis [15]. The model is governed by the following population balance equation

$$
\frac{\partial n(x, t)}{\partial t}+G(t) \frac{\partial n(x, t)}{\partial x}=0, \quad(x, t) \in \mathbb{R}_{+}^{2},
$$

with initial and boundary conditions:

$$
\begin{aligned}
& n(x, 0)=n_{0}(x) \\
& n(0, t)=\frac{R_{n}(t)}{G(t)},
\end{aligned}
$$

where $n(x, t), R_{n}(t), G(t), x$ and $t$ represent crystal size density, nucleation rate, growth rate, crystal size and time, respectively.

The growth rate is independent of crystal size and given by:

$G(t)=\frac{M_{s}}{2 \rho_{s}} K_{c} \eta\left(C(t)-C_{s a t}(t)\right)^{J}$,

where $M_{s}, \rho_{s}$ and $J$ are molar mass of crystal, density of the solute of crystal and constant exponent, respectively. The variables $C(t)$ and $C_{\text {sat }}(t)$ represent the solute concentration and saturated solute concentration, respectively. $\eta$ denotes the effectiveness factor relating the actual mass flux of solid integrated in the crystal structure to the maximum theoretical flux that would be integrated in the absence of diffusive limitation [16]. The variable $\eta$ is the solution of the following equation

$\frac{K_{c}}{K_{d}}\left(C(t)-C_{s a t}(t)\right)^{J-1} \eta+\eta^{1 / J}-1=0$,

where $K_{d}$ and $K_{c}$ are positive constants, denoting global mass transfer coefficient and growth coefficient, respectively. The variable
$C_{\text {sat }}(t)$ is a function of the crystallizer temperature and defined by Van't Hoff formula (see $[14,17]$ )

$C_{s a t}(t)=a_{s a t} \exp \left(\frac{-\Delta H_{f}}{R T_{c r}(t)}\right)$,

where $a_{s a t}, \Delta H_{f}, R$ and $T_{c r}(t)$ are the saturation constant, the fusion enthalpy, the universal constant of perfect air and the crystallizer temperature, respectively.

In the batch crystallizer, the total mole of solute in the suspension is constant. According to the mole balance, the variable $C(t)$ is given by the algebraic relation

$C(t)=\frac{\left(1-\left(M_{S} / \rho_{S}\right) C_{S}(0)\right) C(0)+C_{S}(0)-C_{S}(t)}{1-\left(M_{S} / \rho_{S}\right) C_{S}(t)}$.

The variable $C_{s}(t)$ is the concentration of solid in the suspension defined by

$C_{s}(t)=\frac{K_{v} \rho_{s}}{M_{s}} \int_{0}^{\infty} x^{3} n(x, t) d x$,

where $K_{v}$ is the shape factor. When the crystal is assumed to be spherical, $K_{v}$ is equal to $\pi / 6$. We notice that, at supersaturated state, $C(t)>C_{\text {sat }}(t)$ and hence growth rate $G(t)$ is always positive and $\eta \in(0$, 1 ). Given $C(t)$ and given $C_{s a t}(t), \eta$ can be computed by solving (5).

The nucleation rate $R_{n}(t)$ can be expressed as sum of primary nucleation $R_{n_{1}}(t)$ and secondary nucleation $R_{n_{2}}(t)$ (see [18] and [14]), i.e.

$R_{n}(t)=R_{n_{1}}(t)+R_{n_{2}}(t)$.

The primary nucleation part is given by

$R_{n_{1}}(t)=a_{n_{1}} \exp \left(\frac{-b_{n_{1}}}{\ln ^{2}\left(C(t) / C_{\text {sat }}(t)\right)}\right)$,

and represents the kinetics of the crystal formation when no crystal is inserted into the solution, where $a_{n_{1}}$ and $b_{n_{1}}$ are primary nucleation constant parameters. The secondary nucleation part is given by

$R_{n_{2}}(t)=K_{n_{2}}\left(C(t)-C_{s a t}(t)\right)^{I_{n 2}} C_{s}(t)^{J_{n 2}}$,

and represents the kinetics of the crystal formation attributable to the influence of the existing crystals in the solution, where $K_{n_{2}}, I_{n 2}$ and $J_{n 2}$ are the secondary nucleation parameters which are positive constants.

The heat balance is described by

$\sum_{i=1}^{3} C p_{i} n_{i}(t) \frac{d T_{c r}(t)}{d t}=-\Delta H_{c} V_{T} \frac{d C_{s}(t)}{d t}-U A\left(T_{c r}(t)-T_{j}(t)\right)$,

where $C p_{1}, C p_{2}$ and $C p_{3}$ are the molar heat capacity of the solid, solute in the solution and the solvent, respectively, $n_{1}, n_{2}$ and $n_{3}$ denote the mole number of the solid, solute in the solution and the solvent, respectively, and $\Delta H_{c}, V_{T}, U, A$ and $T_{j}$ are crystallization enthalpy, the total suspension volume, the overall heat transfer coefficient, contact surface through the jacket wall and jacket temperature, respectively. In practice, the jacket temperature $T_{j}(t)$ is considered as control variable for the process.

The CSD can be also characterized by its moments:

$\mu_{i}(t)=\int_{0}^{\infty} x^{i} n(x, t) d x, \quad i=0,1,2, \ldots$

The moments from zero order to third order represent the total number, the total size, the total surface and the total volume of the crystals per unit volume of suspension, respectively. 
By differentiating these moments, we obtain the following ODE system:

$\frac{d \mu_{i}(t)}{d t}=i G(t) \mu_{i-1}(t), \quad i=1,2, \ldots$

$\frac{d \mu_{0}(t)}{d t}=R_{n}(t)$,

where $G(t)$ and $R_{n}(t)$ have been defined in (4) and (9).

Since these equations form a closed system, we can compute the dynamic evolution of the system with the energy balance and the molar balance. In particular, the system (14) and (15) for $i=1,2,3$ is decoupled from the rest.

The control strategy is elaborated based on cooling the crystallizer temperature to obtain the supersaturation such that the nucleation and growth of the crystals take place. Hence, we assume that the jacket temperature $T_{j}(t)$ is our control input variable and that $T_{c r}(t), C(t), C_{s}(t)$ are our measurable output variables [1]. The controlled output is the crystal size distribution $n(x, t)$.

\section{Discretized model}

Much effort has been invested to develop appropriate numerical schemes for crystallization process simulation. Among these numerical techniques, we can cite: the moment method which consists, by finding a condition of closure, to solve the moments system and to reconstruct the crystal size distribution [19-21]; the finite difference method which consists to approximate the continuous system by discrete system and to study the variation of the state variable at each node [14]; the finite volume method which studies the conservation law of each discretized volume instead of the node [22]; the finite element method which approximates the solution of the PDE with piecewise low order polynomials [23]; and the method of characteristics studying the variation of the state variable along the characteristic curves and proposing a discretization strategy based on the finite differences method [24].

Among these methods, the finite differences method (FDM) is widely used to simulate such processes. The advantages of this method are that it is easy to implement and that we get directly a nice structure from the discretized model. However, the main disadvantage is that we need numerous meshes to get an accurate numerical simulation. Furthermore, a finer mesh will increase the computational time and numerical oscillations which may affect the solution accuracy and the schema stability $[25,26]$. Hence, to eliminate these problems, we choose the method of characteristics (MOC) $[13,24]$.

\subsection{Method of characteristics}

The model described by the PBE is discretized by the method of characteristics as presented in [27] and [24]. From the definition, along a characteristic curve the total derivative of $n(x, t)$ with respect to $t$ is given by

$\frac{d n(x, t)}{d t}=\frac{\partial n(x, t)}{\partial t}+\frac{\partial n(x, t)}{\partial x} \frac{d x}{d t}$.

Since the characteristic curve defined in $x t$-plane satisfies the relation $d x / d t=G(t)$ and from Eq. (1), the variation of $n(x, t)$ along the characteristics curve is given by

$\frac{d n(x, t)}{d t}=\frac{\partial n(x, t)}{\partial t}+\frac{\partial n(x, t)}{\partial x} G(t)=0$.

Hence, the variation of $n(x, t)$ along a characteristics curve is reduced to two ordinary differential equations

$\frac{d n(x, t)}{d t}=0$

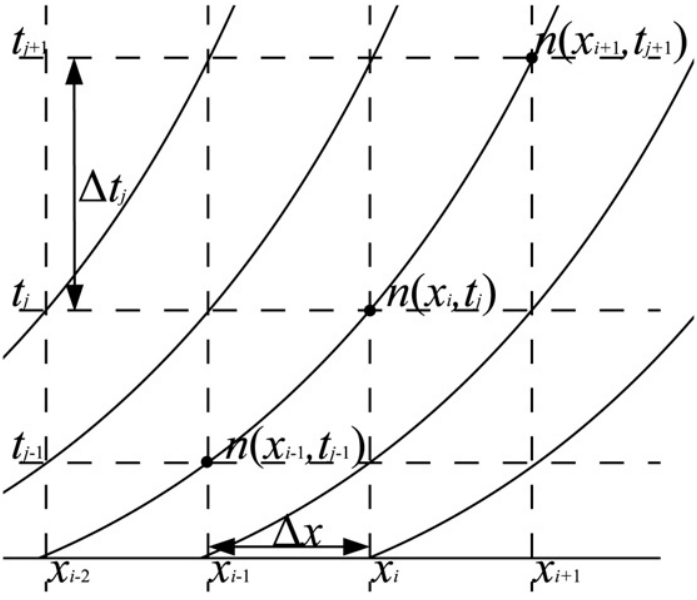

Fig. 1. The method of characteristics in $x t$-plane.

$\frac{d x}{d t}=G(t)$

Since the growth rate $G(t)$ is independent of $x$, the characteristic curves are parallel along the direction of $x$ in the $x t$-plane. Hence, the crystal size is discretized with the fixed step $\Delta x$, and the mesh is created so that the characteristics curves meet mesh nodes at every time step. Consequently, the problem is reduced to find the time step $\Delta t_{j-1}$ beginning at $t_{j-1}$ as illustrated in Fig. 1.

Let $X=\left\{x_{0}, x_{1}, x_{2}, \ldots, x_{N}\right\}$ denote the discrete size set and let $\tilde{n}\left(t_{j}\right)$ be the discretized state

$\tilde{n}\left(t_{j}\right)=\left[n\left(x_{0}, t_{j}\right), n\left(x_{1}, t_{j}\right), \cdots, n\left(x_{N}, t_{j}\right)\right]^{T}$.

We assume that $x_{N}$ is equal to or greater than the biggest crystal size during the process, the crystal is formed at neglected size (i.e. $x_{0}=0$ ), and $\forall x_{i}, x_{i-1} \in X$, a fixed step $\Delta x=x_{i}-x_{i-1}$ is used to discretize the crystal size between 0 and $x_{N}$. Then the discretized model is described by

$\tilde{n}\left(t_{j}\right)=A \tilde{n}\left(t_{j-1}\right)+B \frac{R_{n}\left(t_{j}\right)}{G\left(t_{j}\right)}$,

where

$A=\left[\begin{array}{ccccc}0 & \cdots & \cdots & \cdots & 0 \\ & & & & \\ 1 & \ddots & & & \vdots \\ 0 & \ddots & \ddots & & \vdots \\ \vdots & \ddots & \ddots & \ddots & \vdots \\ 0 & \cdots & 0 & 1 & 0 \\ t_{j} & \\ & =t_{j-1}+\int_{(j-1) \Delta x}^{j \Delta x}\end{array}\right], \quad B=\left[\begin{array}{c}1 \\ 0 \\ \vdots \\ 0 \\ 0\end{array}\right]$

In the discretized model, the concentration of solid $C_{S}$ in (8) is obtained by trapezoidal numerical integration as follows

$C_{s}\left(t_{j}\right)=\frac{K_{v} \rho_{s} \Delta x}{2 M_{S}} \sum_{i=0}^{N-1}\left(x_{i}^{3} n\left(x_{i}, t_{j}\right)+x_{i+1}^{3} n\left(x_{i+1}, t_{j}\right)\right)$.

Notice that, as drawn in Fig. 1, the CSD is constant along the diagonal mesh nodes or $n\left(x_{i}, t_{j}\right)=n\left(x_{i-1}, t_{j-1}\right)$. The time equation (20) can be solved by using the following moment equations

$\frac{d t}{d x}=\frac{1}{G(t)}, \quad \frac{d \mu_{3}}{d x}=3 \mu_{2}, \quad \frac{d \mu_{2}}{d x}=2 \mu_{1}, \quad \frac{d \mu_{1}}{d x}=\mu_{0}, \quad \frac{d \mu_{0}}{d x}=\frac{R_{n}(t)}{G(t)}$. 
Table 1

Parameters used in the simulations.

\begin{tabular}{lll}
\hline Parameter & Unit & Value \\
\hline$M_{s}$ & $\mathrm{~kg} \cdot \mathrm{mol}^{-1}$ & $146.14 \times 10^{-3}$ \\
$\rho_{s}$ & $\mathrm{~kg} \cdot \mathrm{m}^{-3}$ & 1360 \\
$V_{T}$ & $\mathrm{~m}^{3}$ & $0.3 \times 10^{-3}$ \\
$J$ & $\mathrm{Dimensionless}$ & 2.00 \\
$K_{c}$ & $\mathrm{~mol}{ }^{1-J} \cdot \mathrm{m}^{3 J-2} \cdot \mathrm{s}^{-1}$ & $1.57 \times 10^{-2}$ \\
$K_{d}$ & $\mathrm{~m} \cdot \mathrm{s}^{-1}$ & $0.85 \times 10^{-3}$ \\
$K_{v}$ & $\mathrm{Dimensionless}$ & $\pi / 6$ \\
$a_{n_{1}}$ & {$[\mathrm{nb}] \cdot \mathrm{m}^{-3} \cdot \mathrm{s}^{-1}$} & $1.5 \times 10^{12}$ \\
$b_{n_{1}}$ & $\mathrm{Dimensionless}$ & 1.063 \\
$K_{n_{2}}$ & {$[\mathrm{nb}] \cdot \mathrm{m}^{3\left(I_{2}+n_{2}-1\right)}$} & $1.44 \times 10^{3}$ \\
$I_{n_{2}}$ & $\mathrm{~s} \cdot \mathrm{mol}^{\left(I_{n}+n_{2}\right)}$ & 1.968 \\
$J_{n_{2}}$ & $\mathrm{Dimensionless}$ & 1 \\
$a_{s a t}$ & $\mathrm{Dimensionless}$ & $7.492 \times 10^{-6}$ \\
$R$ & $\mathrm{~mol} \cdot \mathrm{m}^{-3}$ & 8.314 \\
$\Delta H_{f}$ & $\mathrm{~J} \cdot \mathrm{K}^{-1} \cdot \mathrm{mol}^{-1}$ & $32,424.6$ \\
$\Delta H_{C}$ & $\mathrm{~J} \cdot \mathrm{mol}^{-1}$ & $-48,000$ \\
$C_{p_{1}}$ & $\mathrm{~J} \cdot \mathrm{mol}^{-1}$ & 372 \\
$C_{p_{2}}$ & $\mathrm{~J} \cdot \mathrm{K}^{-1} \cdot \mathrm{mol}^{-1}$ & 75.33 \\
$C_{p_{3}}$ & $\mathrm{~J} \cdot \mathrm{K}^{-1} \cdot \mathrm{mol}^{-1}$ & 75.33 \\
$U$ & $\mathrm{~J} \cdot \mathrm{K}^{-1} \cdot \mathrm{mol}^{-1}$ & 1000 \\
$A$ & $\mathrm{~W} \cdot \mathrm{m}^{-2} \cdot \mathrm{K}^{-1}$ & $2.2 \times 10^{-2}$ \\
\hline & $\mathrm{m}{ }^{2}$ & \\
\hline
\end{tabular}

\subsection{Parameter sensitivity}

In practice, the nucleation parameters and the growth parameters are obtained from experimental results in the ideal environment condition. However the parameters of nucleation kinetic are sensitive to the environment and hence it is difficult to accurately measure the nuclei number and consequently the parameters are known with uncertainty. Therefore it is necessary to analyze the influence of these parameters. To do so, we simulate the process with different nucleation parameters denoted $\theta=\left[a_{n_{1}}, b_{n_{1}}, K_{n_{2}}, I_{n_{2}}\right]$. The three cases $\theta, \theta+10 \% \theta$ and $\theta-10 \% \theta$ are considered, respectively. The parameters used in the simulation are summarized in Table 1.

The evolutions of the nucleation rate and the final CSD are shown in Figs. 2 and 3, respectively. From the numerical simulations it is seen that the nucleation rate is highly sensitive to the variation of the nucleation parameters, hence the disturbance in these parameters greatly affects the final CSD. In conclusion, the control design for the crystallization process must take into account the nucleation parameter uncertainties.

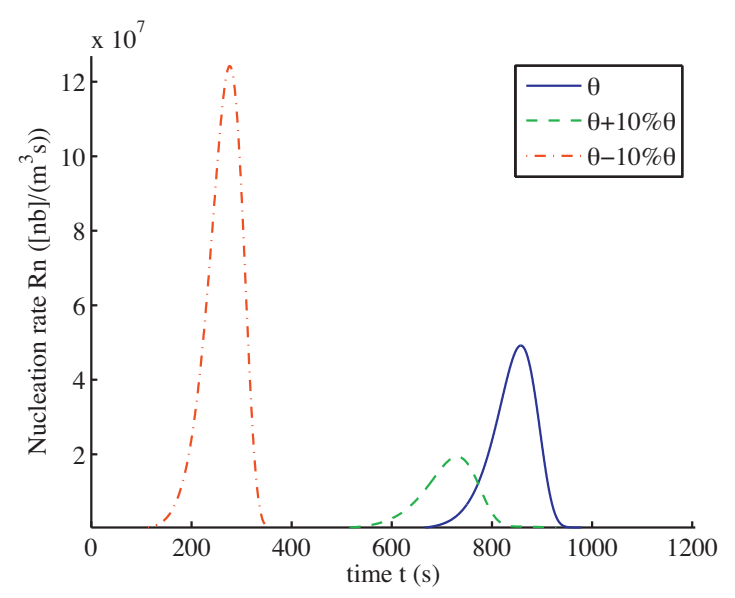

Fig. 2. Evolution of the nucleation rate with different nucleation parameters.

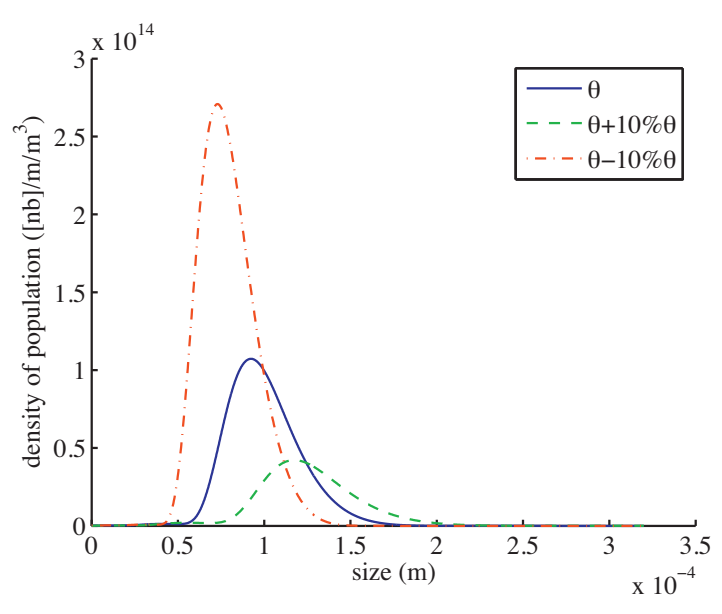

Fig. 3. Final CSD with different nucleation parameters.

\section{Reachability analysis of the batch cooling crystallization}

Without loss of generality, we assume that the crystallizer temperature $T_{c r}(t)$ is completely controllable by the jacket temperature $T_{j}(t)$ (see the heat balance equation (12)). Hence the variable $T_{c r}(t)$ can be used as control variable instead of $T_{j}(t)$.

From (19), it is clear that if the part $R_{n}\left(t_{j}\right) / G\left(t_{j}\right)$ is completely controllable by $T_{c r}(t)$, then we can show that the system is globally controllable. However $R_{n}\left(t_{j}\right)$ and $G\left(t_{j}\right)$ have their physical constraints and the system model is under the supersaturation condition $C(t)>C_{\text {sat }}(t)$. In practice, we have also physical constraint $T_{c r}^{\min } \leq T_{c r}(t) \leq T_{c r}^{\max }$ where $T_{c r}^{\min }$ and $T_{c r}^{\max }$ denote the minimal and the maximal crystallizer temperature, respectively. All these constraints limit the attainable state space through the control of the crystallizer temperature. The reachability study will help us to figure out the limit.

We define the reachability of the crystallization process for the discrete model as follows.

Definition 4.1. $\operatorname{ACSD} n_{d}\left(x_{i}\right), x_{i} \in X$, is called reachable if we can find a sequence of control input $T_{c r}\left(t_{j}\right) \in\left[T_{c r}^{\min }, T_{c r}^{\max }\right], j=1,2, \ldots, K$, such that $n\left(x_{i}, t_{K}\right)=n_{d}\left(x_{i}\right)$ is satisfied for every $x_{i} \in X$.

In other words, a CSD profile $n_{d}\left(x_{i}\right), x_{i} \in X$, is said to be reachable if there is a sequence of control $T_{c r}\left(t_{j}\right), j=1,2, \ldots, K$, which drives the system state to the profile $n_{d}$ at the time $t_{K}$. Now we reconsider the discretized model (19) obtained by the MOC. A simple iteration gives

$n\left(x_{i}, t_{j}\right)=n\left(x_{i-1}, t_{j-1}\right)=n\left(x_{i-2}, t_{j-2}\right)=\cdots$

Hence another representation of the model is given by

$n\left(x_{i}, t_{j}\right)= \begin{cases}n\left(0, t_{j-i}\right)=\frac{R_{n}\left(t_{j-i}\right)}{G\left(t_{j-i}\right)}, & i<j \\ n\left(x_{i-j}, 0\right)=n_{0}\left(x_{i-j}\right), & i \geq j,\end{cases}$

for $i=0,1, \ldots, N$ and $j=0,1, \ldots, K$.

From this representation, we observe that the solution of the model is divided into two parts. The first one determined by the boundary condition depends on the nucleation rate and growth rate which are both related to $T_{c r}$. The second part determined by the initial condition, obviously cannot be controlled by $T_{c r}$. Hence, the desired CSD is expressed in two parts, the controllable part and the initialization part, as

$n_{d}\left(x_{i}\right)= \begin{cases}n_{c}\left(x_{i}\right), & i<K \\ n_{0}\left(x_{i-K}\right), & i \geq K .\end{cases}$ 
The controllable part represents the generation of new crystals. The initialization part is equal to the seeding at the beginning of the batch process.

Back to our objective, according to the desired CSD above, it is sufficient to find a sequence of $T_{c r}\left(t_{j}\right), j=1,2, \ldots, K$, such that, at $t_{K}$

$$
n\left(x_{i}, t_{K}\right)=\frac{R_{n}\left(t_{K-i}\right)}{G\left(t_{K-i}\right)}=n_{c}\left(x_{i}\right), \quad \forall i=0,1, \ldots, K-1 .
$$

From the above equation, the reachability problem is related to the system state vector at $t_{K-i}, \forall i=0,1, \ldots, K-1$. From (4), (10) and (11), we see that both $R_{n}\left(t_{K-i}\right)$ and $G\left(t_{K-i}\right)$ depend on the variables $C\left(t_{K-i}\right), T_{c r}\left(t_{K-i}\right)$ and $C_{s}\left(t_{K-i}\right)$.

$$
\begin{aligned}
& C_{s}\left(t_{K-i}\right)=C_{s}^{d}\left(t_{K-i}\right) \\
& =\frac{K_{v} \rho_{S} \Delta x}{2 M_{S}}\left[\sum_{j=0}^{K-i-2}\left(x_{j}^{3} n_{c}\left(x_{j+i}\right)+x_{j+1}^{3} n_{c}\left(x_{j+i+1}\right)\right)+x_{K-i-1}^{3} n_{c}\left(x_{K-1}\right)+x_{K-i}^{3} n_{0}\left(x_{0}\right)+\sum_{j=K-i}^{N-1}\left(x_{j}^{3} n_{0}\left(x_{j-K+i}\right)+x_{j+1}^{3} n_{0}\left(x_{j-K+i+1}\right)\right)\right], \\
& n\left(., t_{K-i}\right)=\left[\begin{array}{c}
n\left(x_{0}, t_{K-i}\right) \\
n\left(x_{1}, t_{K-i}\right) \\
\vdots \\
n\left(x_{K-i-1}, t_{K-i}\right) \\
n\left(x_{K-i}, t_{K-i}\right) \\
\vdots \\
n\left(x_{N-1}, t_{K-i}\right) \\
n\left(x_{N}, t_{K-i}\right)
\end{array}\right]=\left[\begin{array}{c}
\frac{R_{n}\left(t_{K-i}\right)}{G\left(t_{K-i}\right)} \\
\frac{R_{n}\left(t_{K-i-1}\right)}{G\left(t_{K-i-1}\right)} \\
\vdots \\
\frac{R_{n}\left(t_{1}\right)}{G\left(t_{1}\right)} \\
n_{0}\left(x_{0}\right) \\
\vdots \\
n_{0}\left(x_{N-(K-i)-1}\right) \\
n_{0}\left(x_{N-(K-i)}\right)
\end{array}\right] .
\end{aligned}
$$

On the other hand, if a desired CSD is reachable at $t_{K}$, we can move backward in time and deduce the desired CSD at each time $t_{K-i}$, $i=1, \ldots, K$, as shown in Fig. 1. Using this fact and according to (18) and (26), we obtain the expression of the desired CSD at $t_{K-i}$

$n_{d}\left(., t_{K-i}\right)=\left[\begin{array}{c}n_{d}\left(x_{0}, t_{K-i}\right) \\ n_{d}\left(x_{1}, t_{K-i}\right) \\ \vdots \\ n_{d}\left(x_{K-i-1}, t_{K-i}\right) \\ n_{d}\left(x_{K-i}, t_{K-i}\right) \\ \vdots \\ n_{d}\left(x_{N-1}, t_{K-i}\right) \\ n_{d}\left(x_{N}, t_{K-i}\right)\end{array}\right]=\left[\begin{array}{c}n_{c}\left(x_{i}\right) \\ n_{c}\left(x_{i+1}\right) \\ \vdots \\ n_{c}\left(x_{K-1}\right) \\ n_{0}\left(x_{0}\right) \\ \vdots \\ n_{0}\left(x_{N-(K-i)-1}\right) \\ n_{0}\left(x_{N-(K-i)}\right)\end{array}\right]$.

If, at $t_{K-i-1}$,

$n\left(x_{m}, t_{K-i-1}\right)=n_{d}\left(x_{m}, t_{K-i-1}\right), \quad \forall x_{m} \in X$,

then, at $t_{K-i}$,

$$
\begin{aligned}
n\left(x_{j}, t_{K-i}\right) & =n\left(x_{j-1}, t_{K-i-1}\right) \\
& =n_{d}\left(x_{j-1}, t_{K-i-1}\right) \\
& =n_{d}\left(x_{j}, t_{K-i}\right), \quad j=1,2, \ldots, N .
\end{aligned}
$$

It means that we only need to find a temperature control $T_{c r}\left(t_{K-i}\right)$ such that

$n\left(x_{0}, t_{K-i}\right)=n_{d}\left(x_{0}, t_{K-i}\right), \quad i=1,2, \ldots, K$.

Note that, if at $t_{K}$,

$n\left(x_{m}, t_{K}\right)=n_{d}\left(x_{m}, t_{K}\right), \quad \forall x_{m} \in X$,

then from (19) and going backward in time we show that, at $t_{K-i}$,

$n\left(x_{m}, t_{K-i}\right)=n_{d}\left(x_{m}, t_{K-i}\right), \quad \forall x_{m} \in X, \quad i=1,2, \ldots, K$.

Hence, $C_{S}\left(t_{K-i}\right)$ can be computed from (21) and (27) and given by

where $C_{S}^{d}\left(t_{K-i}\right)$ is the desired solid concentration at $t_{K-i}$ computed from the desired CSD.

The variable $C\left(t_{K-i}\right)$ is computed by using (7) and (30)

$$
C\left(t_{K-i}\right)=C_{d}\left(t_{K-i}\right)=\frac{\left(1-\left(M_{S} / \rho_{s}\right) C_{S}(0)\right) C(0)+C_{S}(0)-C_{s}^{d}\left(t_{K-i}\right)}{1-\left(M_{S} / \rho_{S}\right) C_{S}^{d}\left(t_{K-i}\right)}
$$

where $C_{d}\left(t_{K-i}\right)$ is the desired solute concentration at $t_{K-i}$ computed from the desired CSD.

Hence the control variable $T_{C r}\left(t_{K-i}\right)$ is the only unknown variable in Eq. (25). We should verify if the solution $T_{C r}\left(t_{K-i}\right)$ of (25) really exists. Therefore the reachability problem is reformulated in the proposition below and can be solved by direct computation.

Proposition 4.2. A desired CSD defined by(24)is reachable if the nonlinear algebraic equation(32)has a solution $T_{c r}\left(t_{K-i}\right) \in\left[T_{c r}^{\min }, T_{c r}^{\max }\right]$ :

$\frac{R_{n}\left(t_{K-i}\right)}{G\left(t_{K-i}\right)}=n_{c}\left(x_{i}\right), \quad \forall i=0,1, \ldots, K$,

such that

$$
\begin{aligned}
R_{n}\left(t_{K-i}\right)= & a_{n_{1}} \exp \left(\frac{-b_{n_{1}}}{\ln ^{2}\left(C_{d}\left(t_{K-i}\right) / C_{s a t}\left(t_{K-i}\right)\right)}\right) \\
& +K_{n_{2}}\left(C_{d}\left(t_{K-i}\right)-C_{s a t}\left(t_{K-i}\right)\right)^{I_{n}} C_{s}^{d}\left(t_{K-i}\right)^{I_{2}} \\
G\left(t_{K-i}\right)= & \frac{M_{s}}{2 \rho_{s}} K_{c} \eta\left(C_{d}\left(t_{K-i}\right)-C_{s a t}\left(t_{K-i}\right)\right)^{J} \\
C_{s a t}\left(t_{K-i}\right)= & a_{s a t} \exp \left(\frac{-\Delta H_{f}}{R T_{c r}\left(t_{K-i}\right)}\right),
\end{aligned}
$$

where $C_{S}^{d}\left(t_{K-i}\right) \operatorname{and}_{d}\left(t_{K-i}\right)$ are defined by(30) and (31), respectively.

Proof. If the algebraic equation above has solutions, at each time $t_{K-i}$, a correspondent control $T_{c r}\left(t_{K-i}\right)$ can be found by solving the algebraic equation. Then, we have $R_{n}\left(t_{K-i}\right) / G\left(t_{K-i}\right)=n_{c}\left(x_{i}\right)$ at $t_{K-i}$ From the reachability analysis of the discretized model discussed above, we get $n\left(x_{i}, t_{K}\right)=n_{d}\left(x_{i}\right), \forall x_{i} \in X$ at $t_{K}$.

Remark 4.3. The growth rate $G(t)$ only depends on $T_{c r}(t)$ because $C_{S}(t)$ is known for the desired CSD. Then, if we can find a sequence of the control $T_{c r}\left(t_{j}\right), j=1,2, \ldots, K$, to drive the system to a desired CSD, then we can obtain the growth rate $G(t)$ at each moment $t_{j}$. In this case, we can compute approximately the total process time from (18):

$T \approx \Delta x \sum_{j=0}^{K}\left(G\left(t_{j}\right)\right)^{-1}$. 


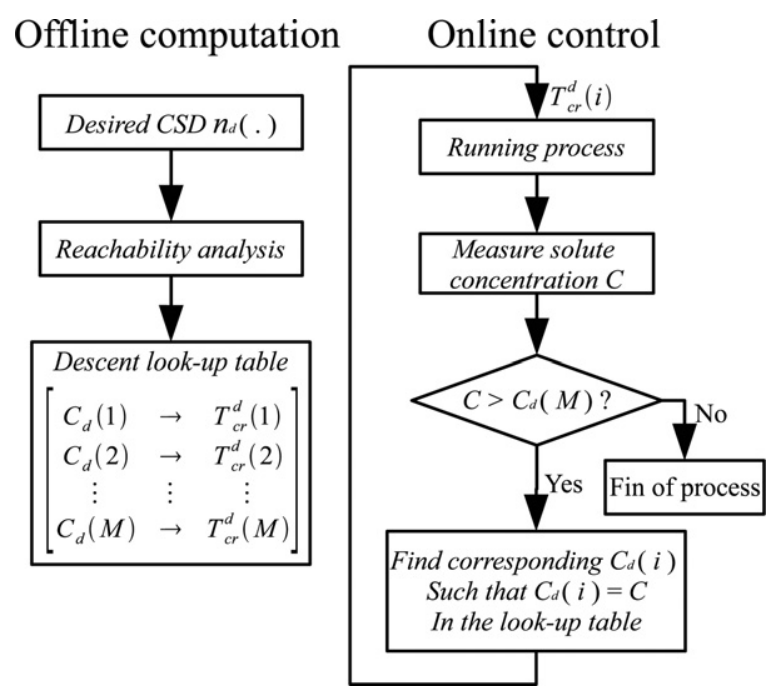

Fig. 4. Feedback control algorithm.

\section{Control design based on the reachability analysis}

\subsection{Closed-loop control design}

Let us discuss the control problem in the ideal conditions (the measurements are not corrupted by any noise and the parameters are exactly known). Given a desired reachable CSD $n_{d}\left(x_{i}\right), \forall x_{i} \in X$, we have a sequence of desired control $T_{c r}^{d}\left(t_{K-i}\right), i=0,1, \ldots, K-1$. To implement our control, it remains to determine $t_{K-i}$ given by Eq. (20) which is difficult to compute. To avoid solving (20), we consider the third moment $\mu_{3}$. Using Eq. (8), we have

$C_{s}=\mu_{3} \frac{K_{v} \rho_{s}}{M_{S}}$

Since, in supersaturation, the growth rate $G(t)$ and the nucleation rate $R_{n}(t)$ (defined in (4) and (9), respectively) are always positives, then $\mu_{3}$ is a monotonically increasing function in time (see (14) and (15)) and so is $C_{s}(t)$. According to (7), it is easy to show that $C(t)$ decreases monotonically with time. From (31), we can compute $C_{d}\left(t_{K-i}\right)$. Using this fact, $T_{c r}^{d}\left(t_{K-i}\right)$ can be replaced by $T_{c r}^{d}\left(C_{d}\left(t_{K-i}\right)\right)$. Therefore, we design a closed-loop control by creating a look-up table between the desired output $C_{d}$ (inside of $t_{i}$ ) and the desired control $T_{c r}^{d}$ as shown in Fig. 4.

\subsection{Feedback control facing model uncertainties}

In this section, we consider the real conditions as described in Section 3 (Fig. 3). Concerned with the closed-loop control algorithm discussed above, the desired control $T_{c r}^{d}(t)$ is computed by using the model without parameter disturbance. However the system can not be driven to the desired CSD by the designed temperature control if parameter disturbance is big. Hence, we propose using another state feedback control to compensate the errors resulted from disturbed parameters.

In the following we assume that $a_{n_{1}}, b_{n_{1}}, K_{n_{2}}$ and $I_{n_{2}}$ are disturbed by the errors $\Delta a_{n_{1}}, \Delta b_{n_{1}}, \Delta K_{n_{2}}$ and $\Delta I_{n_{2}}$. Then the nucleation rates are given by

$$
\begin{aligned}
& \tilde{R}_{n_{1}}(t)=\left(a_{n_{1}}+\Delta a_{n_{1}}\right) \exp \left(\frac{b_{n_{1}}+\Delta b_{n_{1}}}{\ln ^{2}\left(C(t) / C_{s a t}(t)\right)}\right) \\
& \tilde{R}_{n_{2}}(t)=\left(K_{n_{2}}+\Delta K_{n_{2}}\right)\left(C(t)-C_{s a t}(t)\right)^{\left(I_{n 2}+\Delta I_{n_{2}}\right)} C_{s}(t)^{I_{n 2}} .
\end{aligned}
$$

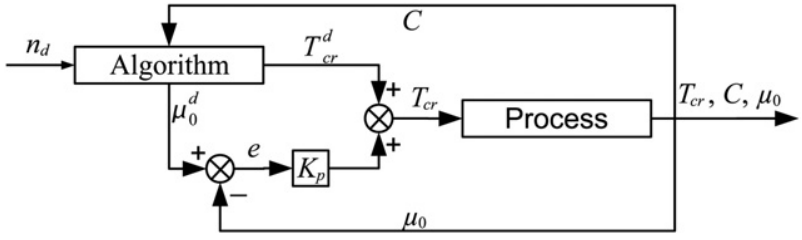

Fig. 5. Feedback control scheme.

The control objective is to design a temperature controller such that, at $t^{\prime}{ }_{K}$, the error

$\sum_{i=0}^{N}\left[n\left(x_{i}, t_{K}^{\prime}\right)-n_{d}\left(x_{i}, t_{K}\right)\right]^{2}$

approaches zero in the presence of parameter uncertainty.

Note that the final time $t_{K}^{\prime}$ of the perturbed model could be different from $t_{K}$ of the ideal model because of the formula (33). The controller design is made based on the discretized model by MOC. From (17) it is clear that the variation of the CSD along the characteristic curve is null. Consequently, to make approach zero the quantity in (36), we propose the following temperature feedback control law

$e=\sum_{i=0}^{N} k_{i}\left[n\left(x_{i}, t_{j}^{\prime}\right)-n_{d}\left(x_{i}, t_{j}\right)\right], \quad \forall j \leq K$

where $k_{i}$ is the static feedback gain.

Unfortunately the variable $n(x, t)$ being not measurable, we can not apply directly the feedback control law (37). We propose to use zero order moment for the feedback design. According to the definition of the zero order moment, the moments $\mu_{0}\left(t_{j}^{\prime}\right)$ and $\mu_{0}^{d}\left(t_{j}\right)$ can be computed by

$\mu_{0}\left(t_{j}^{\prime}\right)=\frac{\Delta x}{2} \sum_{i=0}^{N-1}\left(n\left(x_{i}, t_{j}^{\prime}\right)+n\left(x_{i+1}, t_{j}^{\prime}\right)\right)$,
$\mu_{0}^{d}\left(t_{j}\right)=\frac{\Delta x}{2} \sum_{i=0}^{N-1}\left(n_{d}\left(x_{i}, t_{j}\right)+n_{d}\left(x_{i+1}, t_{j}\right)\right)$.

By using (38) and (39), it is easy to see that

$\mu_{0}\left(t_{j}^{\prime}\right)-\mu_{0}^{d}\left(t_{j}\right)=\frac{\Delta x}{2} \sum_{i=0, i=N}\left[n\left(x_{i}, t_{j}^{\prime}\right)-n_{d}\left(x_{i}, t_{j}\right)\right]+\Delta x \sum_{i=1}^{N-1}\left[n\left(x_{i}, t_{j}^{\prime}\right)-n_{d}\left(x_{i}, t_{j}\right)\right]$.

Let us set $k_{i}$ in (37) as follows

$k_{i}= \begin{cases}\frac{K_{p} \Delta x}{2}, & i=0 ; \\ K_{p} \Delta x, & i=1,2, \ldots, N-1 ; \\ \frac{K_{p} \Delta x}{2}, & i=N,\end{cases}$

where $K_{p}$ is static feedback gain. Using (40) and (41), the feedback control law given by (37) can be written as

$e=K_{p}\left(\mu_{0}\left(t_{j}^{\prime}\right)-\mu_{0}^{d}\left(t_{j}\right)\right), \quad j=1,2, \ldots, K$.

Hence the temperature control with the feedback of the zeroth moment $\mu_{0}$ is proposed to correct the parameter uncertainty. To implement this control, we create a look-up table between $C_{d}, T_{c r}^{d}$ and $\mu_{0}^{d}$. By measuring the solute concentration $C$ and the zeroth moment $\mu_{0}$, the temperature control is given by $T_{c r}=T_{c r}^{d}+K_{p}\left(\mu_{0}^{d}-\right.$ $\left.\mu_{0}\right)$. The corresponding control scheme is illustrated in Fig. 5. 


\subsection{Observer design}

In the proposed control scheme, the feedback control needs the knowledge of the zeroth moment $\mu_{0}$ which is assumed to be measurable. However, in practice, this measurement is expensive and is often corrupted by errors. Nevertheless, we can measure accurately the solid concentration $C_{s}$. Hence, an observer may be designed to estimate the state $\mu_{0}$ using measurements $C_{s}$.

In [28], the author investigated the state and parameter estimation for a class of chemical and biochemical processes, and evaluated the performance of the different state observers in presence of model uncertainties. Here we propose an observer for the moments system constituted of the first four moment equations as follows

$$
\left\{\begin{array}{l}
\frac{d \mu_{3}(t)}{d t}=3 G(t) \mu_{2}(t) \\
\frac{d \mu_{2}(t)}{d t}=2 G(t) \mu_{1}(t) \\
\frac{d \mu_{1}(t)}{d t}=G(t) \mu_{0}(t) \\
\frac{d \mu_{0}(t)}{d t}=R_{n}(t)
\end{array}\right.
$$

As $C_{s}(t)$ is measurable, we can consider that $\mu_{3}(t)$ is also measurable according to (8).

Let us introduce the variable $\tau$ defined by

$\frac{d \tau}{d t}=G(t)$

$\tau(0)=0$.

We assume that the process evolves under the supersaturated condition $C(t)>C_{\text {sat }}(t)$ which implies that $G(t)$ is positive. Hence, $\tau$ is a monotonically increasing function of $t$. From (4) and by assuming that growth rate parameters are exact, we deduced that $G(t)$ is known. Hence, the time transformation $t \rightarrow \tau$ defined in (44) and (45) is invertible. The obtained system with the new time $\tau$ is governed by

$\left[\begin{array}{l}\frac{d \mu_{3}(\tau)}{d \tau} \\ \frac{d \mu_{2}(\tau)}{d \tau} \\ \frac{d \mu_{1}(\tau)}{d \tau} \\ \frac{d \mu_{0}(\tau)}{d \tau}\end{array}\right]=\left[\begin{array}{llll}0 & 3 & 0 & 0 \\ 0 & 0 & 2 & 0 \\ 0 & 0 & 0 & 1 \\ 0 & 0 & 0 & 0\end{array}\right]\left[\begin{array}{c}\mu_{3}(\tau) \\ \mu_{2}(\tau) \\ \mu_{1}(\tau) \\ \mu_{0}(\tau)\end{array}\right]+\left[\begin{array}{c}0 \\ 0 \\ 0 \\ \frac{R_{n}(\tau)}{G(\tau)}\end{array}\right]$,

with the output

$y(\tau)=\mu_{3}(\tau)$.

From (4), (10) and (11), we know that the nucleation rate $R_{n}(\tau)$ and growth rate $G(\tau)$ depend on the output $\mu_{3}(\tau)$ and the input $T_{c r}(\tau)$. By taking into account nucleation parameter uncertainties, the model (46) and (47) is written as follows

$$
\begin{aligned}
& \frac{d \mu(\tau)}{d \tau}=A_{\mu} \mu(\tau)+B_{\mu} g\left(y(\tau), T_{c r}(\tau)\right)+E_{\mu} w(\tau) \\
& y(\tau)=C_{\mu} \mu(\tau) \\
& A_{\mu}=\left[\begin{array}{llll}
0 & 3 & 0 & 0 \\
0 & 0 & 2 & 0 \\
0 & 0 & 0 & 1 \\
0 & 0 & 0 & 0
\end{array}\right], B_{\mu}=\left[\begin{array}{l}
0 \\
0 \\
0 \\
1
\end{array}\right], E_{\mu}=\left[\begin{array}{l}
0 \\
0 \\
0 \\
1
\end{array}\right], C_{\mu}=\left[\begin{array}{llll}
1 & 0 & 0 & 0 \\
& &
\end{array}\right],
\end{aligned}
$$

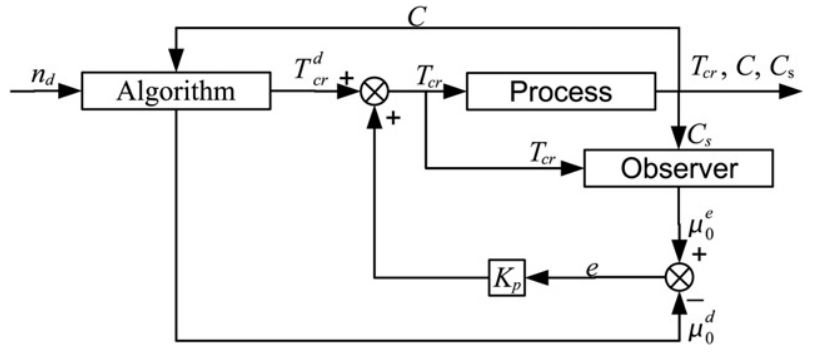

Fig. 6. Feedback control scheme with observer.

where

$$
\begin{aligned}
\mu(\tau)= & {\left[\mu_{3}(\tau), \mu_{2}(\tau), \mu_{1}(\tau), \mu_{0}(\tau)\right]^{T}, } \\
& g\left(y(\tau), T_{c r}(\tau)\right)=\frac{R_{n}\left(y(\tau), T_{c r}(\tau)\right)}{G\left(y(\tau), T_{c r}(\tau)\right)} \\
w(\tau)= & \frac{\tilde{R}_{n}\left(y(\tau), T_{c r}(\tau)\right)}{G\left(y(\tau), T_{c r}(\tau)\right)}-\frac{R_{n}\left(y(\tau), T_{c r}(\tau)\right)}{G\left(y(\tau), T_{c r}(\tau)\right)} .
\end{aligned}
$$

From (48), it is clear that the system is observable (cf. [29]). The aim here is to estimate rapidly the state variables and eliminate the disturbance effect of $w(\tau)$ stemming from parameter uncertainty. Hence the following high gain observer is proposed (for more details see $[29,30])$.

$$
\frac{d \hat{\mu}(\tau)}{d \tau}=A_{\mu} \hat{\mu}(\tau)+B_{\mu} g\left(y(\tau), T_{c r}(\tau)\right)-\Lambda K_{\mu}\left(y(\tau)-C_{\mu}, \hat{\mu}(\tau)\right),
$$

where

$$
\Lambda=\left[\begin{array}{cccc}
\lambda & 0 & 0 & 0 \\
0 & \lambda^{2} & 0 & 0 \\
0 & 0 & \lambda^{3} & 0 \\
0 & 0 & 0 & \lambda^{4}
\end{array}\right], \quad K_{\mu}=\left[\begin{array}{c}
k_{1} \\
k_{2} \\
k_{3} \\
k_{4}
\end{array}\right] .
$$

Then the observer in time area $t$ is governed by

$$
\frac{d \hat{\mu}(t)}{d t}=G(t) A_{\mu} \hat{\mu}(t)+G(t) B_{\mu} g\left(y(t), T_{c r}(t)\right)-G(t) \Lambda K_{\mu}\left(y(t)-C_{\mu} \hat{\mu}(t)\right)
$$

where $\lambda$ is a positive constant sufficiently large and the real constants $k_{1}, k_{2}, k_{3}$ and $k_{4}$ are chosen such that $\left(A_{\mu}+K_{\mu} C_{\mu}\right)$ is a Hurwitz matrix. Integrating the observer in the feedback control loop, we obtain the control scheme shown in Fig. 6.

\section{Results and discussions}

In order to illustrate the proposed control, a batch cooling crystallization model without seedings has been considered. The initial concentration $C(t)$ was assumed to be in the saturated state for the initial temperature. The parameters used in the simulation are listed in Table 1 . The aim is to apply the designed controller such that $T_{c r}$ tracks the desired trajectory $T_{c r}^{d}$ for reaching the desired CSD shown in Fig. 7. This illustration is done on two steps.

(I) The nucleation parameters are exactly known:

Through the Proposition 4.2, a reference table between the desired solute concentration $C_{d}$ and the desired crystallizer temperature $T_{c r}^{d}$ has been established. The variation of $T_{c r}^{d}$ in terms of $C_{d}$ was shown in Fig. 8. Using the control algorithm of Fig. 4, a comparison between the obtained final CSD and the desired CSD is given in Fig. 9. More precisely, by using the relative error defined as

$$
E r r=\frac{\left\|n(x, T)-n_{d}(x)\right\|}{\left\|n_{d}(x)\right\|},
$$




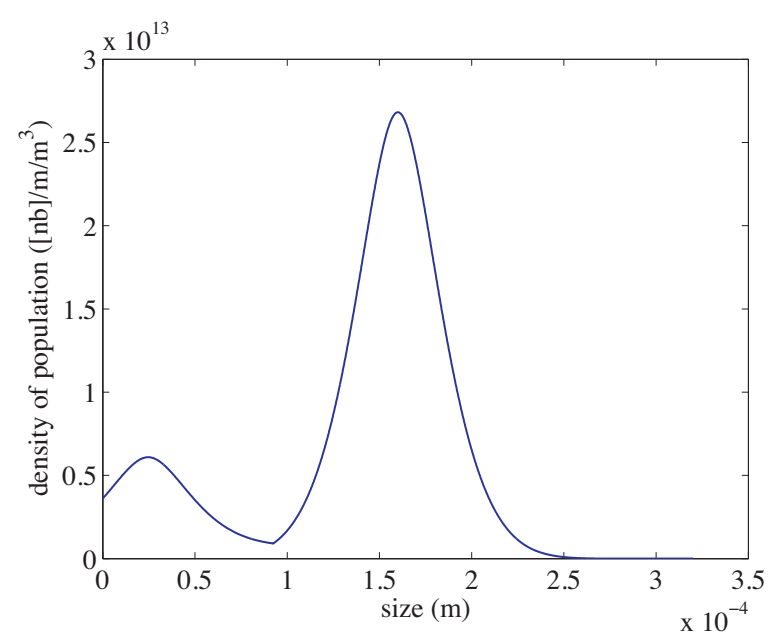

Fig. 7. Desired CSD.

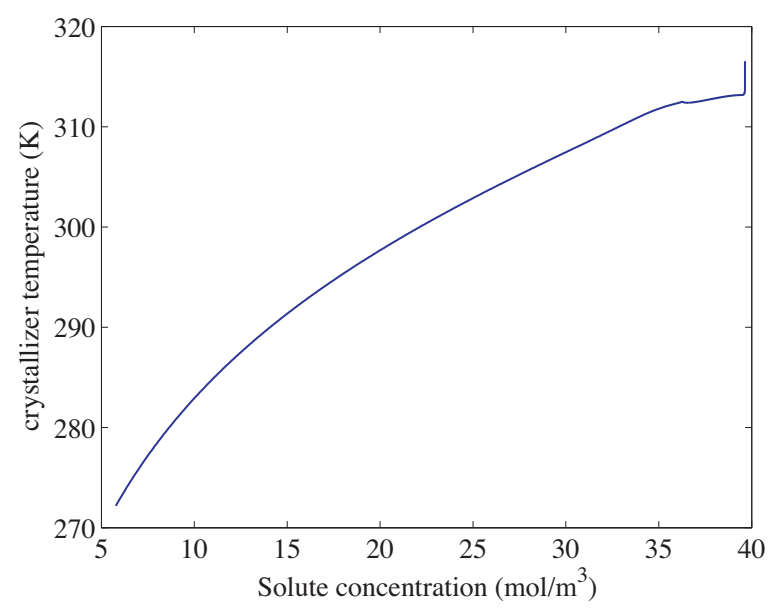

Fig. 8. Desired crystallizer temperature.

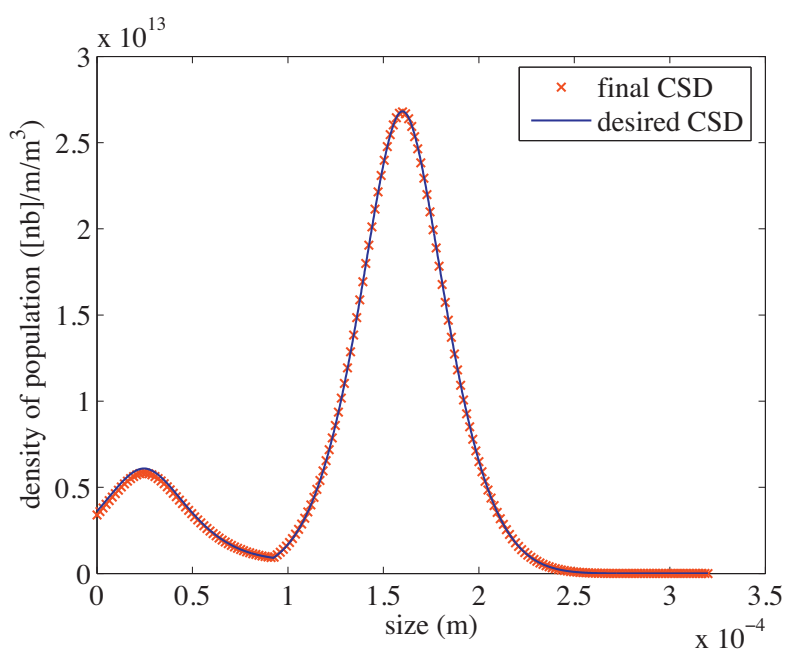

Fig. 9. Final CSD with exact nucleation parameters.

the relative error was evaluated to be $1.3 \%$. From these results we are satisfied of the feasibility and the performances of the proposed controller.

(II) The nucleation parameters are corrupted by errors:

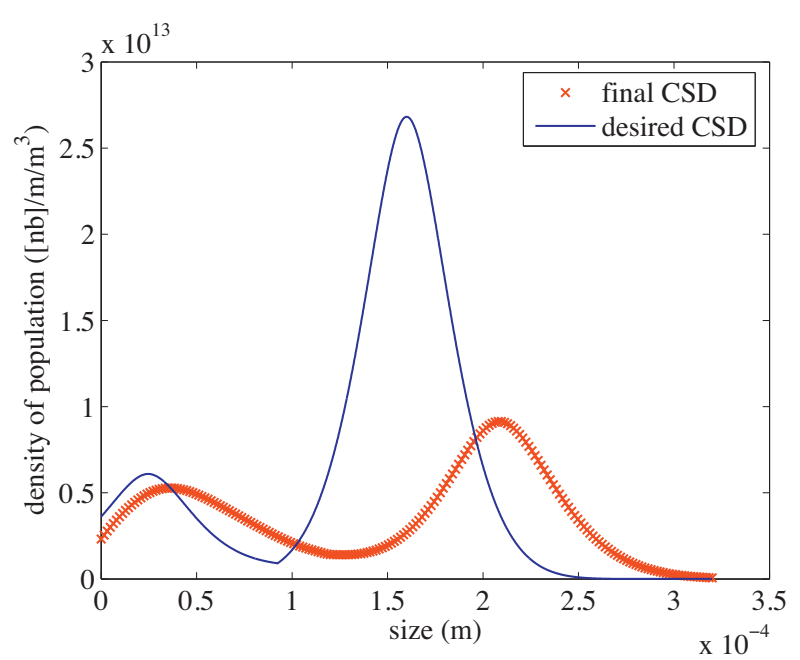

Fig. 10. Final CSD with uncertain nucleation parameters.

We first used the desired control shown in Fig. 8 to drive the system. Simulation results are shown in Fig. 10, and the relative error was equal to $85.6 \%$. We observe that the final CSD was sensitive to the nucleation parameters, and that the two profiles were totally distinct.

Now we use the control scheme with the correction of $\mu_{0}$ shown in Fig. 5, with the corrector gain $K_{p}=-5 \times 10^{-6}$. The final CSD obtained is shown in Fig. 11 . The relative error is $0.88 \%$. From these results, we see that the corrector has efficiently compensated the errors caused by the uncertain nucleation parameters.

Now, we illustrate the global control scheme given by Fig. 6 . Using the estimated $\mu_{0}$ and a corrector gain $K_{p}=-5 \times 10^{-6}$, the final CSD is shown in Fig. 12 and the relative error is $1.47 \%$. From these results, we see that the corrector using the state feedback and the high gain observer has efficiently compensated the errors caused by the uncertain nucleation parameters. However, notice that the controller's performances can be damaged if the measures used for the observer design is corrupted by noise. Usually such a problem may be solved by filtering action.

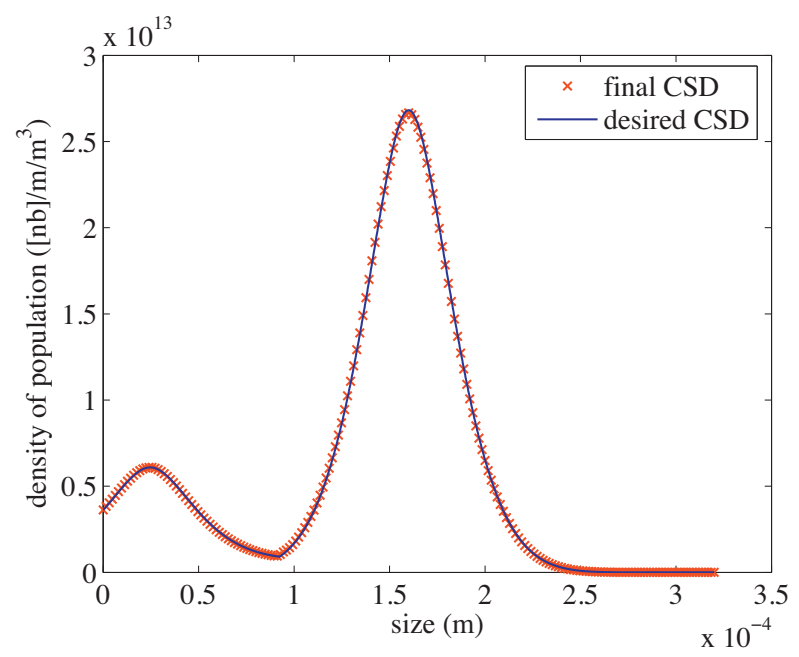

Fig. 11. Final CSD with uncertain nucleation parameters corrected by $\mu_{0}$. 


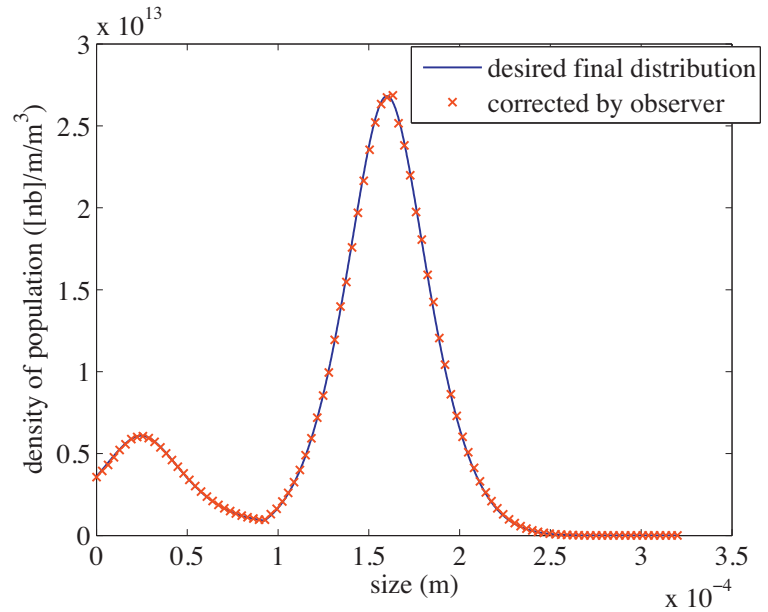

Fig. 12. Final CSD with uncertain nucleation parameters corrected by estimated $\mu_{0}$.

\section{Conclusions}

In this paper we have investigated the reachability of desired crystal size distributions by temperature control for the batch process. A control scheme based on the reachability analysis has been designed to give a temperature trajectory which produces the desired crystal size distribution at the end of the batch. Furthermore the sensitivity of the final CSD to the nucleation parameters has been analyzed and it is concluded that, in this case, the computed control could hardly reach the desired CSD in the presence of parameter uncertainty. An output feedback control law to correct this uncertainty has been proposed and evaluated by simulations. By using the observability canonical form, a high gain observer has been designed to estimate the state which was difficult to measure. The high gain observer has been incorporated into the design of the output controller. The simulation results have demonstrated good performances and efficient correction of the designed controller facing parameter uncertainty.

\section{Acknowledgements}

The authors would like to acknowledge the support by grant "IPAPI 2007-2010 (programme BLAN 07-1/_202691)" of the Agence Nationale de la Recherche (Improving the Properties of Active Pharmaceutical Ingredients). They are also grateful to the AE and anonymous referees for their valuable feedback and comments.

\section{References}

[1] R.D. Braatz, Advanced control of crystallization processes, Annual Reviews in Control 26 (1) (2002) 87-99.

[2] M. Fujiwara, Z.K. Nagy, J.W. Chew, R.D. Braatz, First-principles and direct design approaches for the control of pharmaceutical crystallization, Journal of Process Control 15 (5) (2005) 493-504.
[3] D.L. Ma, R.D. Braatz, Worst-case analysis of finite-time control policies, IEEE Transactions on Control Systems Technology 9 (5) (2001) 766-774.

[4] Z.K. Nagy, R.D. Baraatz, Worst-case and distributional robustness analysis of finite-time control trajectories for nonlinear distributed parameter systems, IEEE Transactions on Control Systems Technology 11 (5) (2003) 694-704.

[5] S.H. Chung, D.L. Ma, R.D. Braatz, Optimal seeding in batch crystallization, The Canadian Journal of Chemical Engineering 77 (3) (1999) 590-596.

[6] Q. Hu, S. Rohani, D. Wang, A. Jutan, Optimal control of a batch cooling seeded crystallizer, Powder Technology 156 (2-3) (2005) 170-176.

[7] G.P. Zhang, S. Rohani, On-line optimal control of a seeded batch cooling crystallizer, Chemical Engineering Science 58 (9) (2003) 1887-1896.

[8] Z.K. Nagy, R.D. Braatz, Robust nonlinear model predictive control of batch processes, AIChE Journal 49 (7) (2003) 1776-1786

[9] Z.K. Nagy, Model based robust control approach for batch crystallization product design, Computers \& Chemical Engineering 33 (10) (2009) 1685-1691.

[10] D. Semino, W.H. Ray, Control of systems described by population balance equations. I. Controllability analysis, Chemical Engineering Science 50 (11) (1995) 1805-1824.

[11] A. Kalbasenka, A. Huesman, H. Kramer, O. Bosgra, Controllability analysis of industrial crystallizers, in: Proceedings of the 12th International Workshop on Industrial Crystallization, Halle, Germany, 2005, pp. 157-164.

[12] U. Vollmer, J. Raisch, Control of batch crystallization-a system inversion approach, Chemical Engineering and Processing 45 (10) (2006) 874-885.

[13] T.-T. Li, B.-P. Rao, Exact boundary controllability for quasi-linear hyperbolic systems, SIAM Journal on Control and Optimization 41 (6) (2003) 1748-1755.

[14] T. Bakir, S. Othman, G. Fevotte, H. Hammouri, Nonlinear observer of crystalsize distribution during batch crystallization, AIChE Journal 52 (6) (2006) 2188-2197.

[15] W.L. McCabe, Crystal growth in aqueous solutions. I. Theory, Industrial \& Engineering Chemistry 21 (1) (1929) 30-33.

[16] J. Garside, The concept of effectiveness factors in crystal growth, Chemical Engineering Science 26 (9) (1971) 1425-1431.

[17] J.-P. Klein, R. Boistelle, J. Dugua, Cristallisation industrielle-aspects théoriques, Techniques de l'Ingénieur J3 (J1500) (1994) J1500.1-J1500.21.

[18] A. Mersmann, Crystallization Technology Handbook, 2nd ed., Marcel Dekker, 2001 .

[19] E. Aamir, Z.K. Nagy, C.D. Rielly, T. Kleinert, B. Judat, Combined quadrature method of moments and method of characteristics approach for efficient solution of population balance models for dynamic modeling and crystal size distribution control of crystallization processes, Industrial \& Engineering Chemistry Research 48 (18) (2009) 8575-8584.

[20] A. Giaya, R.W. Thompson, Recovering the crystal size distribution from the moment equations, AIChE Journal 50 (4) (2004) 879-882.

[21] D.L. Marchisio, J.T. Pikturna, R.O. Fox, R.D. Vigil, A.A. Barresi, Quadrature method of moments for population-balance equations, AIChE Journal 49 (5) (2003) 1266-1276.

[22] R.J. LeVeque, Finite Volume Methods for Hyperbolic Problems, Cambridge University Press, Cambridge, 2002.

[23] A.W. Mahoney, F.J. Doyle III, D. Ramkrishna, Inverse problems in population balances: growth and nucleation from dynamic data, AIChE Journal 48 (5) (2002) 981-990.

[24] K.-I. Sotowa, K. Naito, M. Kano, S. Hasebe, I. Hashimoto, Application of the method of characteristics to crystallizer simulation and comparison with finite difference for controller performance evaluation, Journal of Process Control 10 (2-3) (2000) 203-208

[25] A. LePourhiet, Résolution numérique des équations aux dérivées partielles: une première approche, Cépadues Édition, 1988.

[26] A. William, Numerical Methods for Partial Differential Equations, Academic Press, Inc., 1992.

[27] B.A. Finlayson, Numerical Methods for Problems with Moving Fronts, Ravenna Park Publishing, Inc., Seattle, 1992, ISBN 0963176501

[28] D. Dochain, State and parameter estimation in chemical and biochemical processes: a tutorial, Journal of Process Control 13 (8) (2003) 801-818.

[29] J.P. Gauthier, H. Hammouri, S. Othman, A simple observer for nonlinea systems-applications to bioreactors, IEEE Transaction on Automatic Control 37 (6) (1992) 875-880.

[30] G. Besançon, Nonlinear Observers and Applications, Springer-Verlag, 2007. 